\title{
Lingual Hematoma: A Rare Cause of Upper Airway Obstruction
}

\author{
${ }^{1}$ Eurico Costa, ${ }^{2}$ Hugo Estibeiro, ${ }^{3}$ Miguel Magalhães
}

\begin{abstract}
Introduction: The authors present a case report of a patient with a lingual hematoma treated in the otolaryngology department of Portuguese Institute of Oncology, Lisbon, followed by a brief literature review.

Case report: A case of a 43 years old female with a diagnosis of acute lymphoblastic leukemia since 2007, submitted to allograft bone marrow transplantation in 2008 at the Portuguese Oncology Institute, Lisbon. With diagnosis of recurrence since May 2011, she was hospitalized in October 2011 , due to a lower gastrointestinal bleeding in the context of a cytomegalovirus colitis associated with pancytopenia. During hospitalization, the patient experienced a tonic-clonic seizure. Less than 12 hours after this episode, the patient developed a massive lingual hematoma. Despite exuberant macroglossia and tongue proptosis, the patient showed no signs of active bleeding or severe dyspnea and was submitted to conservative medical treatment.
\end{abstract}

Discussion: Lingual hematoma has many causes, being trauma and hemorrhagic dyscrasias among the most frequent. The first therapeutic objective should be evaluated and guaranted upper airway patency. Once the airway is secured, hemorrhage and hematoma should be treated according to its etiology. This can be achieved with conservative medical therapy, intervention radiology or surgery.

Conclusion: Lingual hematoma is as rare as potentially fatal. Prompt recognition and management is critical for a favorable outcome.

Keywords: Lingual hematoma, Upper airway obstruction, Causes, Clinical management.

How to cite this article: Costa $E$, Estibeiro $H$, Magalhães $M$. Lingual Hematoma: A Rare Cause of Upper Airway Obstruction. Int J Head Neck Surg 2015;6(1):41-44.

Source of support: Nil

Conflict of interest: None

\section{INTRODUCTION}

Lingual hematoma is a rare but potentially fatal cause of upper airway obstruction. The tongue is essentially a muscular organ, with functions in speech, swallowing

\footnotetext{
${ }^{1}$ Department of Otolaryngology, Professor Dr Fernando Fonseca Hospital, Lisbon, Portugal

${ }^{2,3}$ Department of Otolaryngology, Portuguese Institute of Oncology, Lisbon, Portugal
}

Corresponding Author: Eurico Costa, Department of Otolaryngology, Professor Dr Fernando Fonseca Hospital, Lisbon Portugal Phone: 00351932545226, e-mail: euricosta@gmail.com and taste. ${ }^{1}$ It is a highly vascularized structure with arterial supply arising essentially from the lingual artery, an external carotid branch. ${ }^{1}$ Lingual hematoma may have a fulminant evolution, making prompt recognition and management critical for a favorable outcome.

Authors present a case report of a patient with a lingual hematoma attended at the otorhinolaryngology department of Portuguese Institute of Oncology Francisco Gentil, Lisbon.

\section{CASE REPORT}

A 43-year-old female patient treated by the bone marrow transplantation unit (BMTU) since 2007, for acute lymphoblastic leukemia secondary to chronic myeloid leukemia. In 2008, the patient underwent unrelateddonor allograft bone marrow transplant with complete remission, maintaining since immunosuppression with tacrolimus. In 2009 and again in 2011, hematologic relapse was detected and treated with dasatinib and ponatinib respectively.

In October 2011, she was admitted to BMTU with gastrointestinal bleeding secondary to cytomegalovirus colitis and pancytopenia and began therapy with foscarnet, piperacillin-tazobactam, vitamin $\mathrm{K}$ and intensive transfusion support. On the second day, a tonic-clonic seizure episode was observed which reverted without neurological deficits. Nearly 12 hours after this event, the patient developed an impressive looking, violet colored macroglossia associated with lingual proptosis, marked speech limitation, dysphagia and drooling but without dyspnea or dysphonia. Despite absence of trismus, the patient could not close her mouth due to significant tongue volume, augmentation, which projected beyond the dental arcade (Figs 1 and 2). There was no active bleeding or visible laceration on the tongue mucosa. Laryngoscopy revealed tongue base and vallecula involvement with hemorrhagic suffusion at the arytenoid level. The vocal cords were mobile, symmetrical and without lesions, with a normal caliber glottal lumen (Figs 3 and 4). Laboratorial values worth mentioning: hemoglobin: $10.0 \mathrm{~g} / \mathrm{dl}$ (N 12-15), platelet count: $22 \times 10^{3} / \mu \mathrm{l}$ (N 150-400); PT-INR 1.40 (N 0.9-1.1); aPTT 22.8s (N 26-34); fibrinogen $0.79 \mathrm{~g} / 1$ (N 1.5-4.0), D-dimers $25180 \mathrm{ng} / \mathrm{ml}$ ( $\mathrm{n}<278)$. 
Magnetic resonance imaging (MRI) was performed to exclude intracranial pathology showing the extensive lingual hematoma (Figs 5 to 7 ).

As there were no signs or symptoms of either airway obstruction or active bleeding, in addition to patient refusal of more aggressive airways control procedures, a conservative medical approach was adopted (methylprednisolone $30 \mathrm{mg}$ bid, platelet and red blood cells concentrates, fresh frozen plasma, fibrinogen and vitaminK). However, informed consent for a potential urgent tracheotomy was obtained.

Remarkable clinical improvement was observed within 48 hours with clear reduction in tongue volume, obviating the need for other measures. The lingual hematoma resolved completely over the next couple of weeks.

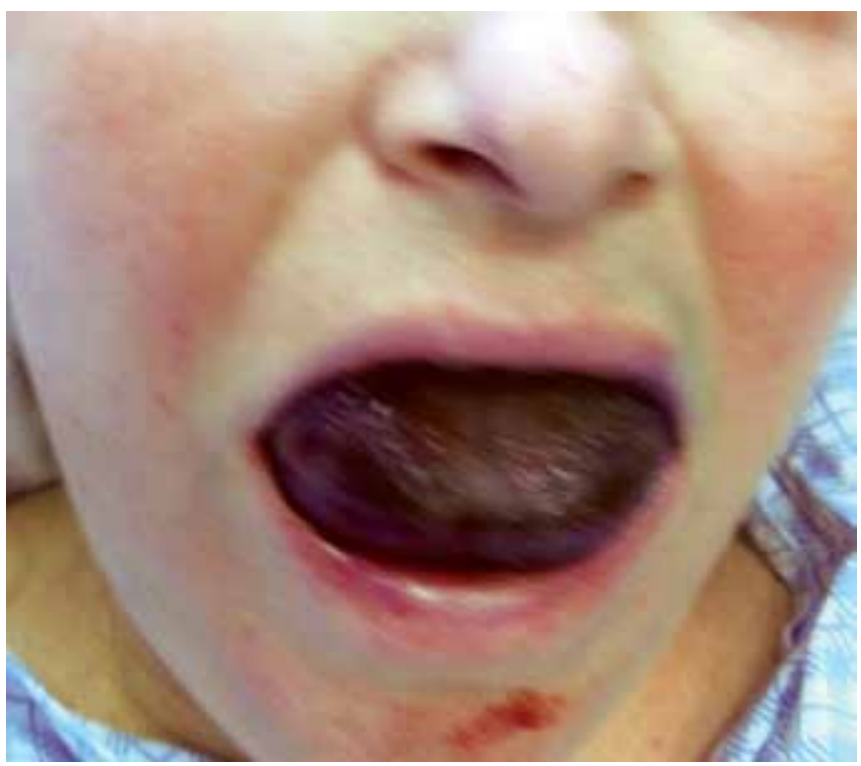

Fig. 1: Massive lingual hematoma preventing mouth closure

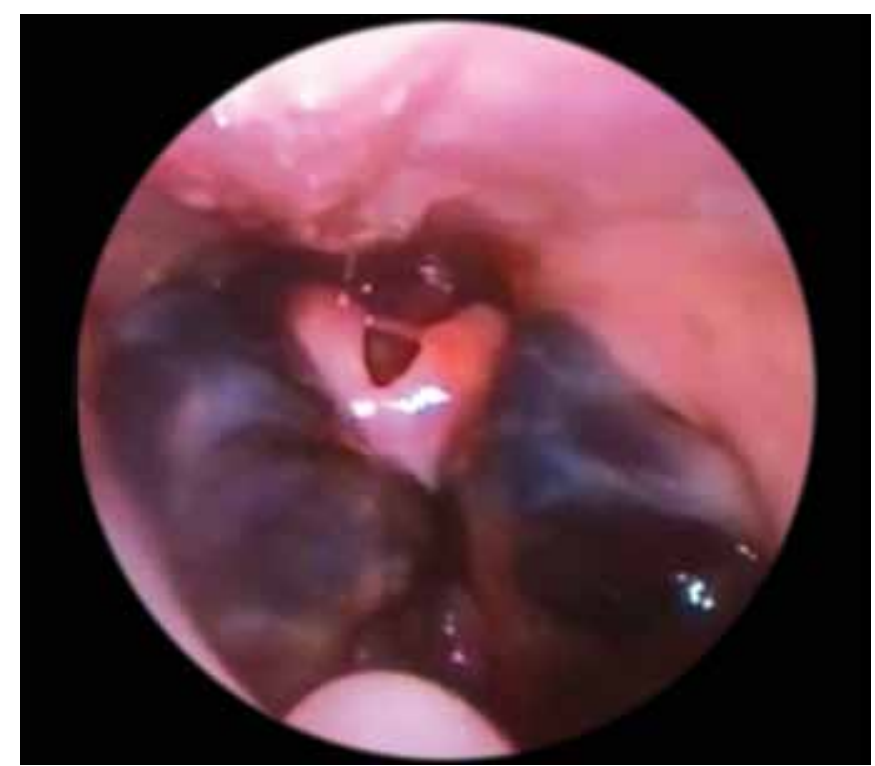

Fig. 3: Flexible nasofibrolaryngoscopy demonstrating involvement of the tongue base and vallecula

\section{DISCUSSION}

There are multiple causes of lingual hematoma: dengue fever, trauma, vascular malformations, thrombocytopenia and coagulopathies, anticoagulant and thrombolytic therapy, etc. ${ }^{2-8}$ In our case report, the probable cause was an association of hemorrhagic predisposition (pancytopenia) with trauma (possible tongue bite during seizure, although no injuries were detected).

Lingual hematoma has the potential for quick obstruction of the upper airway due to tongue's vast vascularization. The increased lingual volume displace it in a posterior and cephalic direction thus blocking the airway. ${ }^{9}$ Bleeding may extend to the sublingual and submental space with a 'pseudo-Ludwig' manifestation. ${ }^{10}$

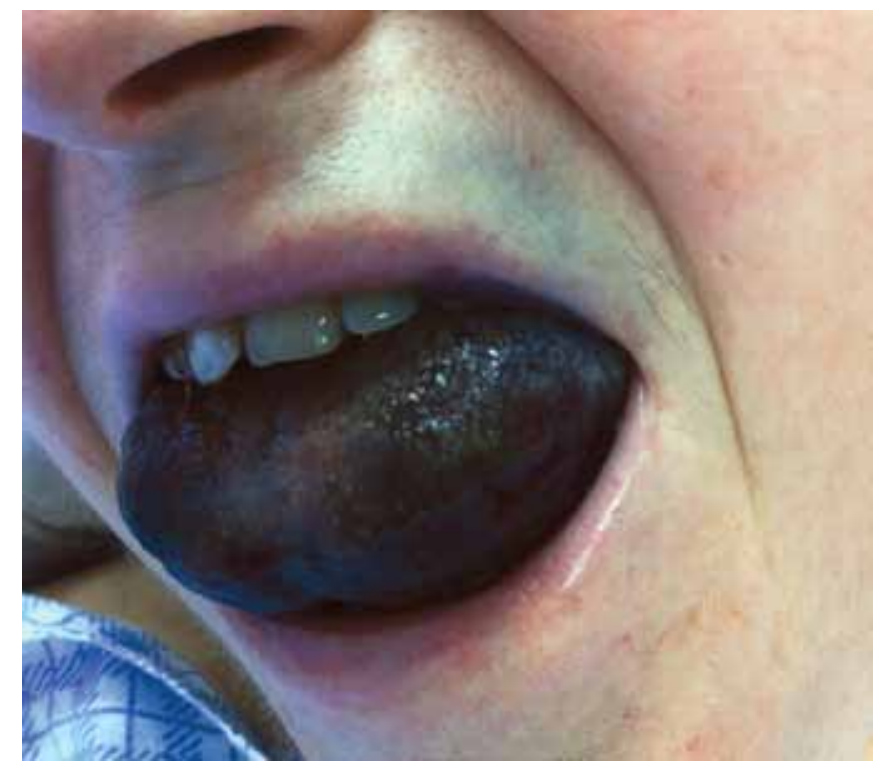

Fig. 2: Lingual hematoma, lateral perspective showing the increased tongue volume and proptosis

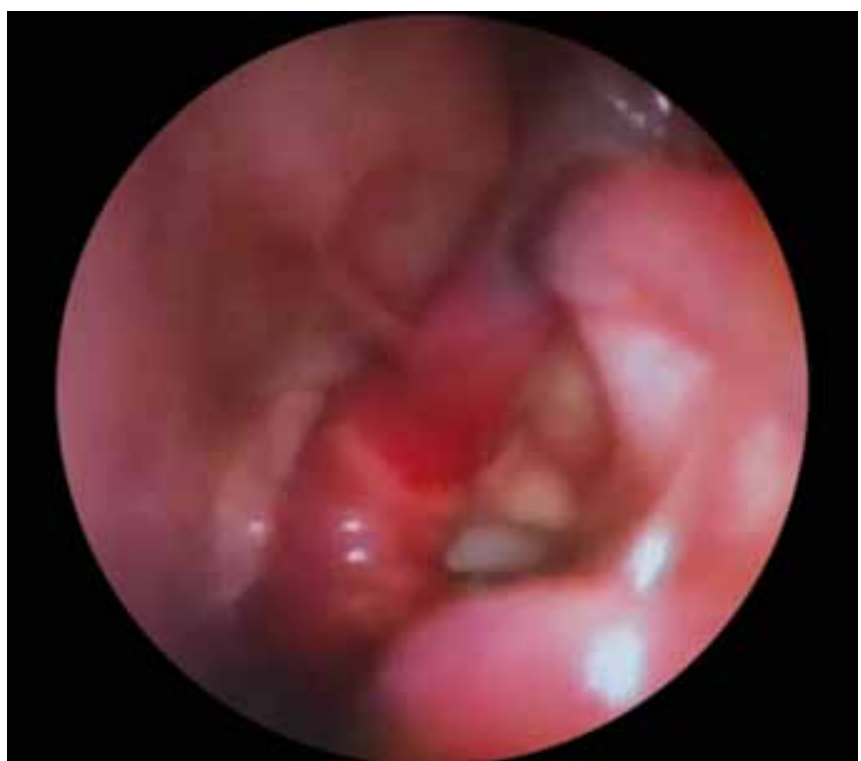

Fig. 4: Flexible nasofibrolaryngoscopy: hemorrhagic suffusion at the arytenoid level, vocal cords mobile, symmetrical and without lesions, with a normal caliber glottal lumen 
The first objective of treatment should be guarantee airway safety. Orotracheal intubation is usually impossible in patients with a hematoma large enough to obstruct the airway. ${ }^{3}$ Awake nasal fiberoptic intubation should be the first option. Last resort, life-saving, emergency cricothyroidotomy can be performed, allowing to ventilate the patient while a safer airway is obtained; tracheotomy is the most efficient long-term solution to secure the airway. ${ }^{3,11}$

Once the airway is secured, the treatment focus switches to hemostasis and etiologic assessment. Most cases associated with bleeding disorders, iatrogenic anticoagulant or thrombolytic therapy can be successfully treated with conservative medical therapy. ${ }^{3}$ Indication to stop anticoagulant or antiaggregant therapy in cases

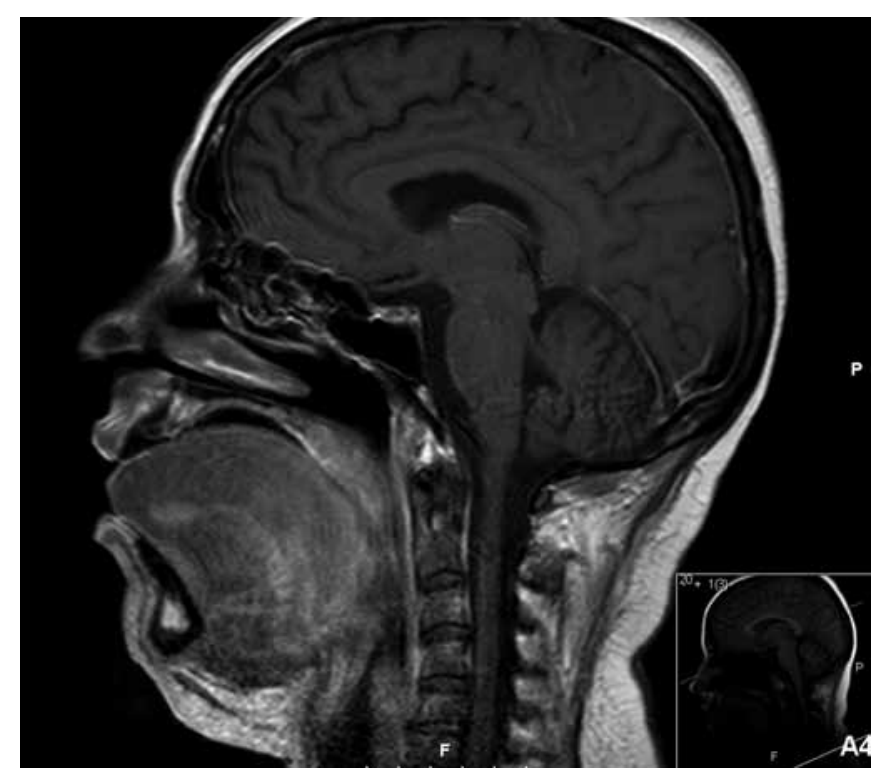

Fig. 5: Magnetic resonance imaging, sagittal view, showing cephalic and posterior tongue displacement due to increased volume, jeopardizing upper airway permeability

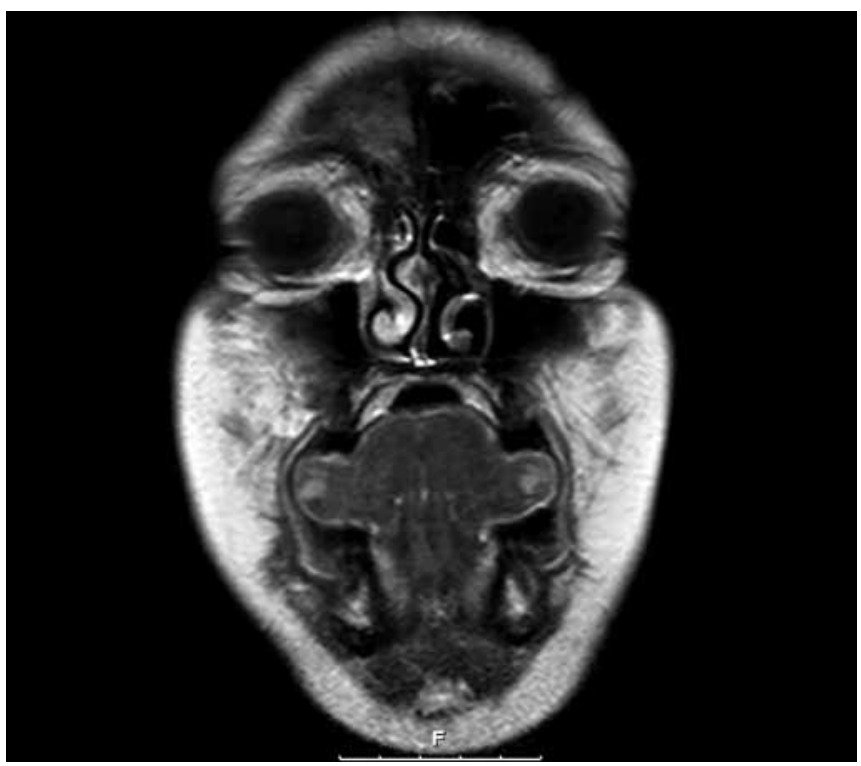

Fig. 6: Magnetic resonance imaging, coronal view, exhibiting enlargement and modeling of the tongue by the upper and lower teeth of coronary heart disease remains a controversial issue and benefits $v s$ risks should be weighed in individual basis. $^{5,8}$

In cases with active bleeding, usually of traumatic origin, hemostasis can be obtained by interventional radiology or ligation of injured vessels by transoral or cervical surgical exploration. Local control with direct pressure, local tongue suture or oral packing is usually inadequate. ${ }^{3}$ Transoral surgical exploration often allows identification and ligation of the injured vessel. It should be noted; however, that surgical drainage of the hematoma should not be attempted as bleeding usually occurs deep in the intrinsic muscles and not in potential spaces between fáscias. ${ }^{12}$

In patients with active bleeding or hematoma expansion in which none of the above measures were successful, ligation of cervical portion of the lingual artery should be attain. ${ }^{3}$ This procedure requires knowledge of the neck anatomy as well as experience in cervical dissection. The lingual artery can be ligated in the carotid or submandibular triangle. ${ }^{13,14}$ Classically, three spaces are described where the cervical portion of lingual artery can be found: Beclard triangle, in the carotid triangle, and Lesser and Pirogoff triangles, in the submandibular triangle (Fig. 8). ${ }^{13,14}$

In our particular case, thrombocytopenia and probable consumptive coagulopathy associated with CMV colitis played a significant role in the development of lingual hematoma. Thus, attending to the patient will, we decided for medical therapy with intensive transfusion support, corticosteroids and vitamin K, despite knowledge that the triggering mechanism might have been a traumatic tongue bite during seizure. No active

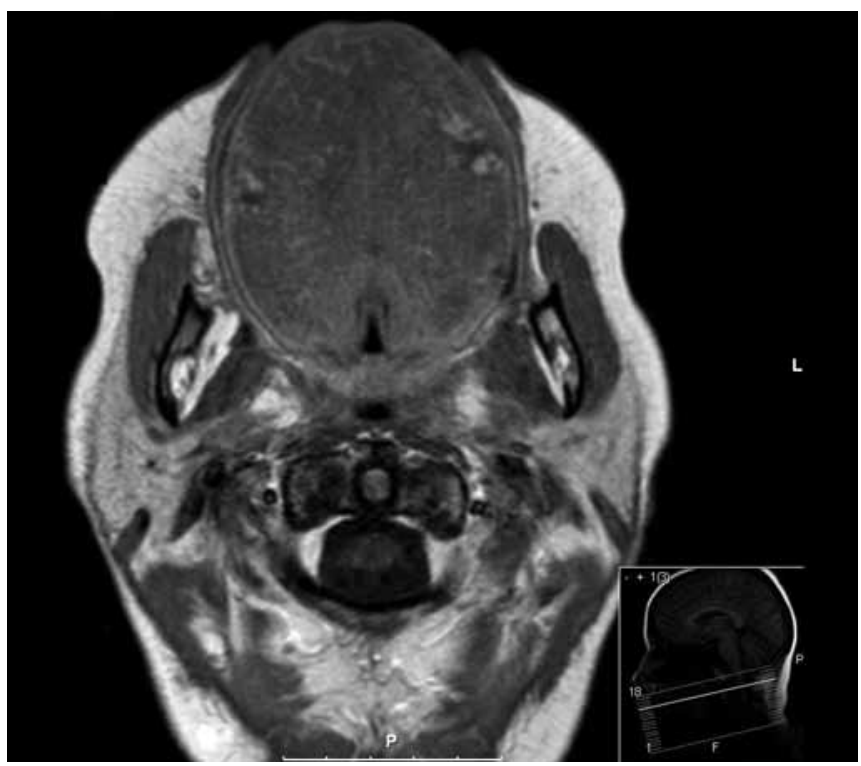

Fig. 7: Magnetic resonance imaging, axial view, highlighting the size and proptosis of the tongue 


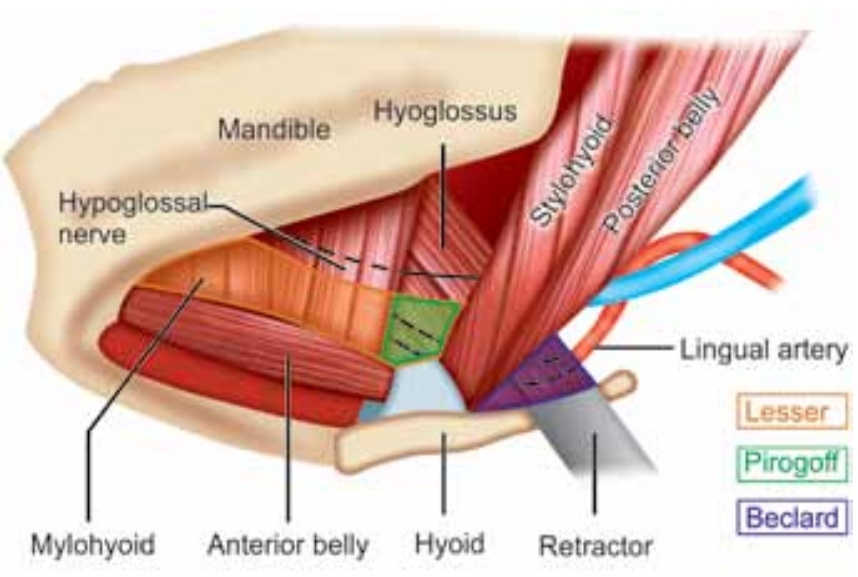

Fig. 8: Anatomical triangles of Beclard (in the carotid triangle), Lesser and Pirogoff (in the submandibular triangle)

bleeding or mucosal lesions were identified, and the lingual hematoma resolved without invasive airway control or surgery.

\section{CONCLUSION}

Lingual hematoma is a phenomenon as rare as potentially fatal. The first concern should be evaluate and guarantee upper airway patency. Once the airway is secured, hemorrhage and hematoma should be treated according to its etiology.

\section{REFERENCES}

1. Berkovitz BK. Anatomy of the mouth and dentition. In: Gleeson. Scott-Brown's Otorhinolaryngology, Head and Neck Surgery. 7th ed. London, Hodder Arnold; 2008. p. 1791-1815.

2. Sarkar J, Mohan C, Misra DN, Goel A. Lingual hematoma causing upper airway obstruction: an unusual manifestation of dengue fever. Asian Pac J Trop Med 2011 May;4(5):412-413.
3. Dhaliwal HS, Dhaliwal SS, Heckel RD, Quereshy FA, et al. Diagnosis and management of upper airway obstruction due to lingual hematoma: report of a case. J Oral Maxillofac Surg 2011 Feb;69(2):558-563.

4. Chase CR, Hebert JC, Farnham JE. Post-traumatic upper airway obstruction secondary to a lingual artery hematoma. J Trauma 1987 Aug;27(8):953-954.

5. Kaya A, Ordu S, Albayrak ES, Aydin M, et al. Lingual hematoma due to streptokinase in a patient with acute myocardial infarction. Am J Emerg Med 2010 Oct;28(8): 985-992.

6. Acar F, Girisgin SA, Cander B, Ozdinc S. Lingual haematoma: a rare complication of usual warfarin dose. Emerg Med J 2008 Jul;25(7):406-410.

7. Getnick GS, Lin SJ, Raviv JR, Walsh WE, et al. Lingual hematoma and heparin-induced thrombocytopenia: a case report. Ear Nose Throat J 2008 Mar;87(3):163-165.

8. Shaps HJ, Snyder GE, Sama AE, Rudolph GS. Airway compromise secondary to lingual hematoma complicating administration of tissue plasminogen activator for acute ischemic stroke. Ann Emerg Med 2001 Oct;38(4):447-449.

9. Kattan B, Snyder HS. Lingual artery hematoma resulting in upper airway obstruction. J Emerg Med 1991;9(6):421-424.

10. Lepore ML. Upper airway obstruction induced by warfarin sodium. Arch Otolaryngol 1976 Aug;102(8):505-506.

11. McGill J. Airway management in trauma: an update. Emerg Med Clin North Am 2007 Aug;25(3):603-622.

12. Song Z, Laggan B, Parulis A. Lingual hematoma treatment rationales: a case report. J Oral Maxillofac Surg 2008 Mar; 66(3):535-539.

13. Homze EJ, Harn SD, Bavitz BJ. Extraoral ligation of the lingual artery: an anatomic study. Oral Surg Oral Med Oral Pathol Oral Radiol Endod 1997 Mar;83(3):321-324.

14. van Es RJ, Thuau H. Pirogoff's triangle revisited: an alternative site for microvascular anastomosis to the lingual artery-a technical note. Int J Oral Maxillofac Surg 2000 Jun; 29(3):207-209. 